\title{
Redefining the Shape of Numbers and Three Forms of Calculation
}

\author{
Ji Peng \\ Department of Electronic Information, Nanjing University, Nanjing, China \\ Email: mcfroo@sina.com
}

How to cite this paper: Peng, J. (2021) Redefining the Shape of Numbers and Three Forms of Calculation. Open Access Library Journal, 8: e7277.

https://doi.org/10.4236/oalib.1107277

Received: March 2, 2021

Accepted: March 23, 2021

Published: March 26, 2021

Copyright (C) 2021 by author(s) and Open Access Library Inc.

This work is licensed under the Creative Commons Attribution International License (CC BY 4.0).

http://creativecommons.org/licenses/by/4.0/

\section{(c) (i) Open Access}

\begin{abstract}
This paper redefines the Shape of numbers, makes it more natural and concise, and the domain of definition is extended to ring. The inconvenient $\mathrm{PCHG}()$ and $\mathrm{PH}()$ are removed. The concept of subsets is also removed. The new definition can be used to calculate $\sum_{n=0}^{N-1} \prod_{i=1}^{M}\left(K_{i}+n \times D_{i}\right)$ $\sum_{n_{i, j}=0}^{j=N-1} \prod_{i=1}^{M}\left(K_{i}+n_{i, j} \times D_{i}\right), \quad n_{i, j} \leq n_{i+1, j} \quad$ or $n_{i, j}=n_{i+1, j} ; K_{i}, D_{i} \in$ ring . Three
\end{abstract} forms corresponding to three calculation methods are obtained. They can be used as a powerful tool for analysis. Some of the conclusions are: 1) Expressions and properties of two kinds of Stirling number, Lah number and Eulerian number; 2) Expression of power sum of natural numbers; 3) Vandermonde identity, Norlund identity; 4) New congruence and new proof of Wilson theorem; 5) $\sum_{n=1}^{P-1} n^{P-2} \equiv 0 M O D P^{2}, P>3$; 6)

$\sum_{C=0}^{C=M-1}(-1)^{M-1-C} \sum_{P M(P S)=M, P B(P S)=C} M I N(P S)=1$.

\section{Subject Areas}

Discrete Mathematics

\section{Keywords}

Shape of Numbers, Calculation Formula, Combinatorics, Congruence, Stirling Number, Eulerian Number

\section{Introduction}

Peng, J. has introduced Shape of numbers in [1] [2] [3] [4]: $\left(I_{1}, I_{2}, \cdots, I_{M}\right)$, $I_{i} \in Z$. There are $M-1$ intervals between adjacent numbers. $I_{i+1}-I_{i} \leq 1$ means continuity; $I_{i+1}-I_{i}>1$ means discontinuity.

Shape of numbers: collect $\left(I_{1}, \cdots, I_{M}\right)$ with the same continuity and discon- 
tinuity at the same position into a catalog, call it a Shape.

A Shape has a min Item: $\left(K_{1}, K_{2}, \cdots\right)$. Use the symbol $P S=[\mathrm{min}$ Item] to represent it.

If $K_{i+1}-K_{i}=D>1$, only $I_{i+1}-I_{i} \geq D$ is allowed.

If $K_{i+1}-K_{i}=D \leq 1$, only $I_{i+1}-I_{i}=D$ is allowed.

The single $\left(I_{1}, \cdots, I_{M}\right)$ is an item. $I_{1} \times \cdots \times I_{M}$ is the product. $I_{i}$ is a factor.

Example 1.1:

$$
\begin{aligned}
& P S=[2,3] \rightarrow(2,3),(3,4),(1000,1001) \in P S \\
& P S=[-3,-1] \rightarrow(-3,-1),(-3,0),(-2,0),(-3,1), \\
& (-2,1),(-1,1),(1000,2007) \in P S \\
& P S=[1,4,4] \rightarrow(1,4,4),(1,5,5),(2,5,5),(1,6,6), \\
& (2,6,6),(3,6,6) \in P S,(3,5,5) \notin P S \\
& P S=[1,4,6,4] \rightarrow(1,4,7,5),(1,5,7,5),(2,5,7,5) \in P S, \\
& (1,4,7,6),(3,5,7,5) \notin P S
\end{aligned}
$$

$P M(P S)=$ Count of factors.

$P B(P S)=$ Count of discontinuities.

$\operatorname{MIN}(P S)=$ Min product: $\operatorname{MIN}([1,2,3])=1 \times 2 \times 3, \quad M I N([1,2,4])=1 \times 2 \times 4$

Basic Shape: $K_{1}=1$ and intervals $=1$ or 2

$B A S E(P S)=B S:$ if (1) $P B(B S)=P B(P S),(2) P M(B S)=P M(P S),(3) B S$ is a Basic Shape, (4) $B S$ has discontinuity intervals at the same positions of $P S$.

$P H(P S)=($ Max Factor $)-1-P B(B S)$

$I D X(P S)=I D X$ of $B S=\{$ Max factor of $B S\}+1=P M(B S)+P B(B S)+1$

$P S=\left[K_{1}, \cdots, K_{M}\right], \quad B S=\left[G_{1}, \cdots, G_{M}\right]$ then $\quad K_{i+1}-K \leq 1 \rightarrow G_{i+1}-G_{i}=1$; $K_{i+1}-K>1 \rightarrow G_{i+1}-G_{i}=2$

Example 1.2:

$$
\begin{aligned}
& P S=[1,2],[1001,1002] \rightarrow B A S E(P S)=[1,2] \\
& P S=[1,3],[-9,4],[1, K>2] \rightarrow B A S E(P S)=[1,3] \\
& P S=[1,3,4],[-8,4,5],[1, K>2, X=K+1] \rightarrow B A S E(P S)=[1,3,4] \\
& P S=[1,3,5],[0,4,9],[1, K>2, X>K+1] \rightarrow B A S E(P S)=[1,3,5] \\
& P S=[1001,1002] \rightarrow P H(P S)=1002-1-0=1001, \operatorname{IDX}(P S)=\operatorname{IDX}([1,2])=3 \\
& P S=[0,4,9] \rightarrow P H(P S)=9-1-2=6, \operatorname{IDX}([0,4,9])=\operatorname{IDX}([1,3,5])=6
\end{aligned}
$$

$\operatorname{SET}(N, P S)=S E T$ of items $\in P S$ in $\left[K_{1}, N-1\right]$, item's max factor $\leq N-1$

[3] introduced the subset: fix some interval of discontinuities.

$S E T(N, P S, P T)=$ Subset of $S E T(N, P S), \operatorname{BASE}(P S)=\left[G_{1}, \cdots, G_{M}\right]$, $P T=\left[T_{1}, \cdots, T_{M}\right]$

$$
=\left\{\begin{array}{l}
T_{i+1}-T_{i}=1: G_{i+1}-G_{i}=1, \text { means } I_{i+1}-I_{i}=K_{i+1}-K_{i} \\
T_{i+1}-T_{i}=1: G_{i+1}-G_{i}=2, \text { means } I_{i+1}-I_{i}=K_{i+1}-K_{i} \\
T_{i+1}-T_{i}=2: G_{i+1}-G_{i}=2, \text { means } I_{i+1}-I_{i}>=K_{i+1}-K_{i}
\end{array}\right.
$$


$P T$ only has the change at $\left(^{*}\right)$. When a change happens, make the interval fixed. $P C H G(P S, P T)=$ Count of change from $B A S E(P S)$ to $P T$

Example 1.3:

$\operatorname{PCHG}([1,3,5],[1,3,5])=0$

$\operatorname{PCHG}([1,3,5],[1,2,4])=\operatorname{PCHG}([1,4,7],[1,2,4])=1$, changed at $T_{1}$

$\operatorname{PCHG}([1,3,5],[1,3,4])=\operatorname{PCHG}([1,4,7],[1,3,4])=1$, changed at $T_{2}$

$\operatorname{PCHG}([1,3,5],[1,2,3])=\operatorname{PCHG}([1,8,10],[1,2,3])=2$, changed at $T_{1}, T_{2}$

$|S E T(N, P S, P T)|=$ Count of items in $S E T(N, P S, P T)$

$S U M(N, P S, P T)=$ Sum of all products in $S E T(N, P S, P T)$

Example 1.4:

$$
\begin{aligned}
& \operatorname{SUM}(6,[1,2,4])=1 \times 2 \times 4+1 \times 2 \times 5+2 \times 3 \times 5 \\
& \operatorname{SUM}(9,[1,4,7])=\operatorname{SUM}(9,[1,4,7],[1,3,5]) \\
&=1 \times 4 \times 7+1 \times 4 \times 8+1 \times 5 \times 8+2 \times 5 \times 8 \\
& \operatorname{SUM}(9,[1,4,7],[1,2,4])=1 \times 4 \times 7+1 \times 4 \times 8+2 \times 5 \times 8
\end{aligned}
$$

[1] [2] [3] [4] came to the following conclusion:

$$
|\operatorname{SET}(N, P S, P T)|=\left(\begin{array}{c}
N-P H(P S)-P C H G(P S, P T)-1 \\
P B(P T)+1
\end{array}\right)
$$

The following uses count of $X \in K$ for count of $\left\{X_{1}, X_{2}, \cdots, X_{M}\right\} \in\left\{K_{1}, K_{2}, \cdots, K_{M}\right\}$

(1.2) Use the form $\left(T_{1}+K_{1}\right)\left(T_{2}+K_{2}\right) \cdots\left(T_{M}+K_{M}\right)=\sum X_{1} X_{2} \cdots X_{M}, \quad X_{i}=T_{i}$ or $K_{i}$.

Don't swap the factors of $X_{1} X_{2} \cdots X_{M}$, then each $X_{1} X_{2} \cdots X_{M}$ corresponds to a expression $=A_{q}\left(\begin{array}{c}N-P H(P S)-P C H G(P S, P T)-1 \\ I D X(P T)-q\end{array}\right), q=$ count of $X \in K$.

$$
\begin{aligned}
& \operatorname{SUM}(N, P S, P T)=\sum A_{q}\left(\begin{array}{c}
N-P H(P S)-P C H G(P S, P T)-1 \\
I D X(P T)-q
\end{array}\right) \\
& A_{q}=\prod_{i=1}^{M}\left(X_{i}+D_{i}\right), \quad D_{i}=\left\{\begin{array}{l}
-m: X_{i}=T_{i}, m=\text { count of }\left\{X_{1}, \cdots, X_{i-1}\right\} \in K \\
+m: X_{i}=K_{i}, m=\text { count of }\left\{X_{1}, \cdots, X_{i-1}\right\} \in T
\end{array}\right.
\end{aligned}
$$

Example 1.5:

$$
\begin{aligned}
P S= & {\left[K_{1}, K_{2} \geq K_{1}+2, K_{3} \geq K_{2}+2\right], \quad B S=B A S E(P S)=[1,3,5], } \\
\rightarrow & I D X(B S)=6, \\
\text { form }= & \left(1+K_{1}\right)\left(3+K_{2}\right)\left(5+K_{3}\right) \\
= & 1 \times 3 \times 5+1 \times 3 \times K_{3}+1 \times K_{2} \times 5+1 \times K_{2} \times K_{3}+K_{1} \times 3 \times 5 \\
& +K_{1} \times 3 \times K_{3}+K_{1} \times K_{2} \times 5+K_{1} \times K_{2} \times K_{3} \\
P= & N-P H(P S)-P C H G(P S, B S)-1 \\
= & N-\left\{K_{3}-1-2\right\}-0-1=N-K_{3}+2
\end{aligned}
$$




$$
\begin{aligned}
\rightarrow & \operatorname{SUM}(N, P S) \\
= & 1 \times 3 \times 5\left(\begin{array}{l}
P \\
6
\end{array}\right)+1 \times 3 \times\left(K_{3}+2\right)\left(\begin{array}{l}
P \\
5
\end{array}\right)+1 \times\left(K_{2}+1\right) \times(5-1)\left(\begin{array}{l}
P \\
5
\end{array}\right) \\
& +1 \times\left(K_{2}+1\right) \times\left(K_{3}+1\right)\left(\begin{array}{l}
P \\
4
\end{array}\right)+K_{1} \times(3-1) \times(5-1)\left(\begin{array}{l}
P \\
5
\end{array}\right) \\
& +K_{1} \times(3-1) \times\left(K_{3}+1\right)\left(\begin{array}{l}
P \\
4
\end{array}\right)+K_{1} \times K_{2} \times(5-2)\left(\begin{array}{l}
P \\
4
\end{array}\right) \\
& +K_{1} \times K_{2} \times K_{3}\left(\begin{array}{l}
P \\
3
\end{array}\right)
\end{aligned}
$$

An item $=\left\{K_{1}+E_{1}, \cdots, K_{M}+E_{M}\right\}, K$ is fixed, $E$ is variable.

A product $=\left(K_{1}+E_{1}\right) \cdots\left(K_{M}+E_{M}\right)=\sum F_{1} F_{2} \cdots F_{M}, F_{i}=E_{i}$ or $K_{i}$

That is, a product can be broken down into $2^{M}$ parts.

Define $S U M_{-} K\left(N, P S, P T, P F=F_{1} \cdots F_{M}\right)=$ Sum of one part, $P F$ indicates the part.

Rewrite 1.2), add \{braces\}:

$$
\begin{aligned}
& \operatorname{SUM}(N, P S, P T)=\sum \prod_{i=1}^{M}\left(X_{i}+D_{i}\right)\left(\begin{array}{c}
A \\
M_{q}
\end{array}\right), \\
& X_{i}+D_{i}=\left\{\begin{array}{l}
\left\{T_{i}-D_{i}\right\}: X_{i}=T_{i}, D_{i}=\text { count of }\left\{X_{1}, \cdots, X_{i-1}\right\} \in K \\
\left\{K_{i}\right\}+\left\{D_{i}\right\}: X_{i}=K_{i}, D_{i}=\text { count of }\left\{X_{1}, \cdots, X_{i-1}\right\} \in T
\end{array}\right.
\end{aligned}
$$

Expand $S U M()$ by $\{$ braces\}:

(1.3) $S U M_{-} K(N, P S, P T, P F)$

$$
=\sum \text { Expansion of } S U M() \text { with same }\left\{K_{i}\right\} \in P F=\sum \prod_{i=1}^{M} Y_{i}\left(\begin{array}{c}
A \\
M_{q}
\end{array}\right)
$$

$$
Y_{i}=\left\{\begin{array}{l}
0: F_{i}=K_{i}, X_{i}=T_{i} \\
K_{i}: F_{i}=K_{i}, X_{i}=K_{i} \\
T_{i}-D_{i}: F_{i}=E_{i}, X_{i}=T_{i}, D_{i}=\text { count of }\left\{\cdots, X_{i-1}\right\} \in K \\
D_{i}: F_{i}=E_{i}, X_{i}=K_{i}, D_{i}=\text { count of }\left\{\cdots, X_{i-1}\right\} \in T
\end{array}\right.
$$

Example 1.6:

Use the example above

$$
\begin{aligned}
& S U M_{-} K\left(\cdots E_{1} E_{2} E_{3}\right)=1 \times 3 \times 5\left(\begin{array}{l}
P \\
6
\end{array}\right)+[1 \times 3 \times 2+1 \times 1 \times(5-1)]\left(\begin{array}{l}
P \\
5
\end{array}\right)+1^{3}\left(\begin{array}{l}
P \\
4
\end{array}\right) \\
& S U M_{-} K\left(\cdots E_{1} E_{2} K_{3}\right)=1 \times 3 \times K_{3}\left(\begin{array}{l}
P \\
5
\end{array}\right)+1 \times 1 \times K_{3}\left(\begin{array}{l}
P \\
4
\end{array}\right) \\
& S U M_{-} K\left(\cdots E_{1} K_{2} E_{3}\right)=1 \times K_{2} \times(5-1)\left(\begin{array}{l}
P \\
5
\end{array}\right)+1 \times K_{2} \times 1\left(\begin{array}{l}
P \\
4
\end{array}\right) \\
& S U M_{-} K\left(\cdots K_{1} E_{2} E_{3}\right)=K_{1} \times(3-1) \times(5-1)\left(\begin{array}{l}
P \\
5
\end{array}\right)+K_{1} \times(3-1) \times 1\left(\begin{array}{l}
P \\
4
\end{array}\right) \\
& S U M_{-} K\left(\cdots E_{1} K_{2} K_{3}\right)=1 \times K_{2} \times K_{3}\left(\begin{array}{l}
P \\
4
\end{array}\right) \\
& S U M_{-} K\left(\cdots K_{1} E_{2} K_{3}\right)=K_{1} \times(3-1) \times K_{3}\left(\begin{array}{l}
P \\
4
\end{array}\right)
\end{aligned}
$$




$$
\begin{aligned}
& S U M \_K\left(\cdots K_{1} K_{2} E_{3}\right)=K_{1} \times K_{2} \times(5-2)\left(\begin{array}{l}
P \\
4
\end{array}\right) \\
& S U M \_K\left(\cdots K_{1} K_{2} K_{3}\right)=K_{1} \times K_{2} \times K_{3}\left(\begin{array}{l}
P \\
3
\end{array}\right)
\end{aligned}
$$

$S U M \_K()$ can explain why $S U M()$ has that strange form:

We can calculate every part of $S U M()$ by some way without the form. There may be complex relationships between the parts, but their sum match a simple form.

If understand this: In 1.2), when $T_{i}$ and $D_{i}$ all increase $L$ times

$$
\begin{aligned}
& K_{1} \times \cdots \times K_{M}+\left(L+K_{1}\right) \times \cdots \times\left(L+K_{M}\right)+\cdots \\
& +\left((N-1) L+K_{1}\right) \times \cdots \times\left((N-1) L+K_{M}\right)
\end{aligned}
$$

$P T=[1 \times L, 2 \times L, \cdots, M \times L]$, can use the form $\left(T_{1}+K_{1}\right) \cdots\left(T_{M}+K_{M}\right)=\sum A_{q}\left(\begin{array}{c}N \\ M+1-q\end{array}\right), q=$ count of $X \in K, 2^{M}$ items in total.

$$
A_{q}=\prod_{i=1}^{M}\left(X_{i}+D_{i}\right), D_{i}=\left\{\begin{array}{l}
-m L: X_{i}=T_{i}, m=\text { count of }\left\{\cdots, X_{i-1}\right\} \in K \\
+m L: X_{i}=K_{i}, m=\text { count of }\left\{\cdots, X_{i-1}\right\} \in T
\end{array}\right.
$$

This paper starts from (1.4), tries to calculate

(1*) $K_{1} \times \cdots \times K_{M}+\left(D_{1}+K_{1}\right) \times \cdots \times\left(D_{M}+K_{M}\right)+\cdots$

$$
+\left((N-1) D_{1}+K_{1}\right) \times \cdots \times\left((N-1) D_{M}+K_{M}\right)
$$

In the process, the concept of Shape is greatly expanded.

The form of 1.2) is obtained by guess. If the correct form is found, the rest is mainly inductive proof. With the forms, we can analyze the expression and coefficient and get a lot of results.

\section{Redefinition}

Change domain from $Z$ to Ring, $K_{i}, D_{i} \in$ Ring . $K_{i}$ no longer compares big or small.

$M$-series:

$$
\begin{aligned}
& \left\{K_{1}, D_{1}+K_{1}, 2 D_{1}+K_{1}, 3 D_{1}+K_{1}, \cdots,(N-1) D_{1}+K_{1}\right\}=\left\{K_{1}+n \times D_{1}\right\} \\
& \left\{K_{2}, D_{2}+K_{2}, 2 D_{2}+K_{2}, 3 D_{2}+K_{2}, \cdots,(N-1) D_{2}+K_{2}\right\}=\left\{K_{2}+n \times D_{2}\right\} \\
& \vdots \\
& \left\{K_{M}, D_{M}+K_{M}, 2 D_{M}+K_{M}, 3 D_{M}+K_{M}, \cdots,(N-1) D_{M}+K_{M}\right\} \\
& =\left\{K_{M}+n \times D_{M}\right\}
\end{aligned}
$$

An item $=\left(I_{1}, I_{2}, \cdots, I_{M}\right), I_{1}$ come from serie1, $I_{2}$ come from serie $2 \ldots$

Use $P S=\left[K_{1}: D_{1}, K_{2}: D_{2}, \cdots, K_{M}: D_{M}\right]$ to represent the Shape.

$\left[K_{1}: 1, K_{2}: 1, \cdots, K_{M}: 1\right]$ is abbreviated as $\left[K_{1}, K_{2}, \cdots, K_{M}\right]$

If $K_{i} \in Z$, the new definition is similar to the old definition and allows $D_{i} \leq 0$.

$S E T(N, P S, P T)=$ Set of some Items come from $M$-series. 


$$
\begin{aligned}
& I_{i}=K_{i}+a \times D_{i}, \quad I_{i+1}=K_{i+1}+b \times D_{i+1}, \\
& P T=\left[T_{1}=1, T_{2}, \cdots, T_{M}\right]=\left\{\begin{array}{l}
T_{i+1}-T_{i}=1: \text { means } a=b \\
T_{i+1}-T_{i}=2, \text { means } a \leq b
\end{array}\right.
\end{aligned}
$$

There is no longer the idea of subsets.

We have tried to extend the domain of $P T$, but when $M>2$, no rules have been found yet.

Basic Shape: $K_{1}=1$ and intervals $=1$ or $2, K_{i} \in N, D_{i}=1$

$P T$ is always a Basic Shape.

$M I N(P S)=$ Min product of a Basic Shape $=\prod K_{i}$

$|S E T(N, P S, P T)|=$ Count of items $\in \operatorname{SET}(N, P S, P T)$

$E N D(N, P S, P T)=$ Set of Items $\in S U M(N, P S, P T)$ and $I_{M}=(N-1) D_{M}+K_{M}$.

$S U M(N, P S, P T)=$ Sum of products in $S E T(N, P S, P T)$

$\operatorname{SUM}(N, P S,[1,2, \cdots, M])$ is abbreviated as $\operatorname{SUM}(N, P S)$

$\operatorname{SUM}(N, \cdots)=\operatorname{Old} \operatorname{Sum}\left(\operatorname{Max}\left(K_{i}\right)+N, \cdots\right), P H()$ and $P C H G()$ are no longer needed.

The old does not allow $\operatorname{SUM}([2,3],[1,3]), \operatorname{SUM}([3,2],[1,3])$. The new it is allowed.

$$
\begin{gathered}
\operatorname{SUM}(N, P S)=(1 *)=\sum_{n=0}^{N-1} \prod_{i=1}^{M}\left(K_{i}+n \times D_{i}\right) \\
\operatorname{SUM}(N, P S,[1,3,5, \cdots, 2 M-1])=\sum \prod_{i=1}^{M}\left(K_{i}+J_{i, j} \times D_{i}\right), \\
J_{1 . i} \leq J_{2, i} \leq \cdots \leq J_{M, i}, \quad J_{i, j} \in[0, N-1] \\
P M(P S)=M ; P B(P T)=\text { Count of discontinuities in } P T \\
I D X(P T)=T_{M}+1=P B(P T)+P M(P T)+1 \\
\text { 2.1) }|\operatorname{SET}(N, P S, P T)|=\left(\begin{array}{c}
N+P B(P T) \\
P B(P T)+1
\end{array}\right)
\end{gathered}
$$

\section{Form 1 of Calculation}

Similar to [4], key points are:

Define $\nabla f(n)=f(n)-f(n-1)$ : if $f(n)=\sum A_{i}\left(\begin{array}{c}N_{i} \\ m_{i}\end{array}\right)$, then

$\nabla f(n)=\sum A_{i}\left(\begin{array}{l}N_{i}-1 \\ m_{i}-1\end{array}\right)$

1) $\sum_{n=0}^{N-1} n\left(\begin{array}{c}n+K \\ M\end{array}\right)=(M+1)\left(\begin{array}{c}N+K \\ M+2\end{array}\right)+(M-K)\left(\begin{array}{c}N+K \\ M+1\end{array}\right)$

By definition:

2) $\sum E N D(N, P S, P T)=\nabla S U M(N, P S, P T)$

3) $\operatorname{SUM}\left(N,\left[P S, K_{M+1}: D_{M+1}\right],\left[P T, T_{M}=T_{M}+1\right]\right)$

$$
=\sum_{n=0}^{N-1}\left(K_{M+1}+n \times D_{M+1}\right) \times \sum E N D(n+1, P S, P T)
$$

4) $\operatorname{SUM}\left(N,\left[P S, K_{M+1}: D_{M+1}\right],\left[P T, T_{M}=T_{M}+2\right]\right)$

$$
=\sum_{n=0}^{N-1}\left(K_{M+1}+n \times D_{M+1}\right) \times S U M(n+1, P S, P T)
$$


$P S=\left[K_{1}: D_{1}, \cdots, K_{M}: D_{M}\right], \quad P T=\left[T_{1}, \cdots, T_{M}\right], \quad P S 1=\left[P S, K_{M+1}: D_{M+1}\right]$

3.1) Use the Form $_{1}=\left(T_{1}+K_{1}\right) \cdots\left(T_{M}+K_{M}\right)=\sum X_{1} \cdots X_{M}$, $\operatorname{SUM}(N, P S, P T)=\sum A_{q}\left(\begin{array}{c}N+P B(P T) \\ I D X(P T)-q\end{array}\right), q=$ count of $X \in K$. $A_{q}=\prod_{i=1}^{M} B_{i}, \quad B_{i}=\left\{\begin{array}{l}\left(T_{i}-m\right) D_{i} ; X_{i}=T_{i}, m=\text { count of }\left\{X_{1}, \cdots, X_{i-1}\right\} \in K \\ K_{i}+m D_{i} ; X_{i}=K_{i}, m=\text { count of }\left\{X_{1}, \cdots, X_{i-1}\right\} \in T\end{array}\right.$

[Proof]

Suppose $\operatorname{SUM}(N, P S, P T)=\sum X_{1} \cdots X_{M}\left(\begin{array}{c}N+P B(P T) \\ I D X(P T)-q\end{array}\right)$

When $P T 1=\left[P T, T_{M+1}=1+T_{M}\right]$

$\operatorname{SUM}(N, P S 1, P T 1)$

$=\sum_{n=0}^{N-1}\left(K_{M+1}+n \times D_{M+1}\right) \times \sum \operatorname{END}(n+1, P S, P T)$

$=\sum_{n=0}^{N-1}\left(K_{M+1}+n \times D_{M+1}\right) \times \nabla S U M(n+1, P S, P T)$

$=\sum_{n=0}^{N-1}\left(K_{M+1}+n \times D_{M+1}\right) \times \sum X_{1} \cdots X_{M}\left(\begin{array}{c}n+P B(P T) \\ I D X(P T)-q-1\end{array}\right) \stackrel{(1)}{\longrightarrow}$

$=\sum X_{1} \cdots X_{M} D_{M+1}(I D X(P T)-q)\left(\begin{array}{c}N+P B(P T) \\ I D X(P T)-q+1\end{array}\right)$

$+\sum X_{1} \cdots X_{M}\left\{K_{M+1}+(I D X(P T)-q-1-P B(P T)) D_{M+1}\right\}\left(\begin{array}{c}N+P B(P T) \\ I D X(P T)-q\end{array}\right)$

$P B(P T 1)=P B(P T), I D X(P T 1)=I D X(P T)+1, I D X(P T)=1+T_{M}=T_{M+1}, I D X(P T)-P B(P T)-1=M$

$\begin{aligned}= & \sum X_{1} \cdots X_{M} D_{M+1}\left(T_{M+1}-q\right)\left(\begin{array}{c}N+P B(P T 1) \\ I D X(P T 1)-q\end{array}\right) \\ & +\sum X_{1} \cdots X_{M}\left\{K_{M+1}+(M-q) D_{M+1}\right\}\left(\begin{array}{c}N+P B(P T 1) \\ I D X(P T 1)-(q+1)\end{array}\right)\end{aligned}$

The previous expression means $X_{M+1}=T_{M+1}$

$M-q=$ Count of $\left\{X_{1} \cdots X_{M}\right\} \in T$. The following expression means $X_{M+1}=K_{M+1}$

$\rightarrow$ Match the Form $\left(T_{1}+K_{1}\right) \cdots\left(T_{M}+K_{M}\right)\left\{T_{M+1}+K_{M+1}\right\}$.

When $P T 1=\left[P T, T_{M+1}=2+T_{M}\right]$

$\operatorname{SUM}(N, P S 1, P T 1)$

$=\sum_{n=0}^{N-1}\left(K_{M+1}+n \times D_{M+1}\right) \times S U M(n+1, P S, P T)$

$=\sum_{n=0}^{N-1}\left(K_{M+1}+n \times D_{M+1}\right) \times \sum X_{1} \cdots X_{M}\left(\begin{array}{c}n+1+P B(P T) \\ I D X(P T)-q\end{array}\right) \stackrel{(1)}{\longrightarrow}$

$=\sum X_{1} \cdots X_{M} D_{M+1}(I D X(P T)-q+1)\left(\begin{array}{c}N+1+P B(P T) \\ I D X(P T)-q+2\end{array}\right)$

$+\sum X_{1} \cdots X_{M}\left\{K_{M+1}+(I D X(P T)-q-1-P B(P T)) D_{M+1}\right\}$

$\times\left(\begin{array}{c}N+1+P B(P T) \\ I D X(P T)-q+1\end{array}\right) \stackrel{P B(P T 1)=P B(P T)+1, I D X(P T 1)=I D X(P T)+2}{\longrightarrow}$ 


$$
\begin{aligned}
= & \sum X_{1} \cdots X_{M} D_{M+1}\left(T_{M+1}-q\right)\left(\begin{array}{c}
N+P B(P T 1) \\
I D X(P T 1)-q
\end{array}\right) \\
& +\sum X_{1} \cdots X_{M}\left\{K_{M+1}+(M-q) D_{M+1}\right\}\left(\begin{array}{c}
N+P B(P T 1) \\
I D X(P T 1)-(q+1)
\end{array}\right)
\end{aligned}
$$

$\rightarrow$ Match the Form $\left(T_{1}+K_{1}\right) \cdots\left(T_{M}+K_{M}\right)\left\{T_{M+1}+K_{M+1}\right\}$.

q.e.d.

The proof process can be extended to ring.

Example 3.1:

$P S=[7: 13,-7.7: 2.2,15:-23], \quad P T=[1,3,5], \quad$ Form $_{1}=(1+7)(3-7.7)(5+15)$

$\operatorname{SUM}(N, P S, P T)$

$=-9867.0\left(\begin{array}{c}N+2 \\ 6\end{array}\right)+1084.6\left(\begin{array}{c}N+2 \\ 5\end{array}\right)+4044.7\left(\begin{array}{c}N+2 \\ 4\end{array}\right)-808.5\left(\begin{array}{c}N+2 \\ 3\end{array}\right)$

$-808.5=7 \times(-7.7) \times 15$

$$
\begin{aligned}
4044.7= & {[(1-0) \times 13] \times(-7.7+2.2 \times 1) \times(15-23 \times 1) } \\
& +7 \times[(3-1) \times 2.2] \times(15-23 \times 1) \\
& +7 \times(-7.7) \times[(5-2) \times(-23)]
\end{aligned}
$$

$1084.6=[(1-0) \times 13] \times[(3-0) \times 2.2] \times(15-23 \times 2)$

$$
\begin{aligned}
& +[(1-0) \times 13] \times(-7.7+2.2 \times 1) \times[(5-1) \times(-23)] \\
& +7 \times[(3-1) \times 2.2] \times[(5-1) \times(-23)]
\end{aligned}
$$

$-9867.0=[(1-0) \times 13] \times[(3-0) \times 2.2] \times[(5-0) \times(-23)]$

$\operatorname{SUM}(2, P S, P T)$

$=7 \times(-7.7) \times 15+7 \times(-7.7-5.5) \times(-8)+20 \times(-5.5) \times(-8)$

$=4044.7\left(\begin{array}{l}4 \\ 4\end{array}\right)-808.5 \times\left(\begin{array}{l}4 \\ 3\end{array}\right)=810.7$

$\operatorname{SUM}(3, P S, P T)$

$=\operatorname{SUM}(2, P S, P T)+7 \times(-7.7-5.5-3.3) \times(-31)$

$+20 \times(-5.5-3.3) \times(-31)+33 \times(-3.3) \times(-31)$

$=1084.6\left(\begin{array}{l}5 \\ 5\end{array}\right)+4044.7\left(\begin{array}{l}5 \\ 4\end{array}\right)-808.5\left(\begin{array}{l}5 \\ 3\end{array}\right)=13223.1$

$\operatorname{SUM}(4, P S, P T)$

$=S U M(3, P S, P T)+7 \times(-7.7-5.5-3.3-1.1) \times(-54)$

$+20 \times(-5.5-3.3-1.1) \times(-54)+33 \times(-3.3-1.1) \times(-54)$

$+46 \times(-1.1) \times(-54)$

$=-9867.0\left(\begin{array}{l}6 \\ 6\end{array}\right)+1084.6\left(\begin{array}{l}6 \\ 5\end{array}\right)+4044.7\left(\begin{array}{l}6 \\ 4\end{array}\right)-808.5\left(\begin{array}{l}6 \\ 3\end{array}\right)=41141.1$ 
Example 3.2:

$$
\begin{aligned}
& P S=\left[\left(\begin{array}{ll}
7 & 3 \\
1 & 2
\end{array}\right):\left(\begin{array}{ll}
1 & 3 \\
4 & 2
\end{array}\right),\left(\begin{array}{ll}
2 & 1 \\
1 & 2
\end{array}\right):\left(\begin{array}{ll}
2 & 3 \\
1 & 2
\end{array}\right)\right], \quad P T=[1,3] \\
& \text { Form }_{1}=\left(1+\left(\begin{array}{ll}
7 & 3 \\
1 & 2
\end{array}\right)\right)\left(3+\left(\begin{array}{ll}
2 & 1 \\
1 & 2
\end{array}\right)\right) \\
& \left(\begin{array}{cc}
17 & 13 \\
4 & 5
\end{array}\right)=\left(\begin{array}{ll}
7 & 3 \\
1 & 2
\end{array}\right)\left(\begin{array}{ll}
2 & 1 \\
1 & 2
\end{array}\right) \\
& \left(\begin{array}{ll}
44 & 70 \\
28 & 38
\end{array}\right)=1 \times\left[\left(\begin{array}{ll}
2 & 1 \\
1 & 2
\end{array}\right)+\left(\begin{array}{ll}
2 & 3 \\
1 & 2
\end{array}\right)\right]\left(\begin{array}{ll}
1 & 3 \\
4 & 2
\end{array}\right)+\left(\begin{array}{ll}
7 & 3 \\
1 & 2
\end{array}\right) \times(3-1)\left(\begin{array}{ll}
2 & 3 \\
1 & 2
\end{array}\right) \\
& \left(\begin{array}{ll}
15 & 27 \\
30 & 48
\end{array}\right)=1 \times\left(\begin{array}{ll}
1 & 3 \\
4 & 2
\end{array}\right) \times 3 \times\left(\begin{array}{ll}
2 & 3 \\
1 & 2
\end{array}\right) \\
& S U M(N, P S, P T)=\left(\begin{array}{cc}
15 & 27 \\
30 & 48
\end{array}\right)\left(\begin{array}{c}
N+1 \\
4
\end{array}\right)+\left(\begin{array}{cc}
44 & 70 \\
28 & 38
\end{array}\right)\left(\begin{array}{c}
N+1 \\
3
\end{array}\right)+\left(\begin{array}{cc}
17 & 13 \\
4 & 5
\end{array}\right)\left(\begin{array}{c}
N+1 \\
2
\end{array}\right) \\
& S U M(2, P S, P T)=\left(\begin{array}{ll}
7 & 3 \\
1 & 2
\end{array}\right)\left(\begin{array}{ll}
2 & 1 \\
1 & 2
\end{array}\right)+\left[\left(\begin{array}{ll}
7 & 3 \\
1 & 2
\end{array}\right)+\left(\begin{array}{ll}
8 & 6 \\
5 & 4
\end{array}\right)\right]\left(\begin{array}{ll}
4 & 4 \\
2 & 4
\end{array}\right) \\
& =\left(\begin{array}{c}
2+1 \\
3
\end{array}\right)\left(\begin{array}{cc}
44 & 70 \\
28 & 38
\end{array}\right)+\left(\begin{array}{c}
2+1 \\
2
\end{array}\right)\left(\begin{array}{cc}
17 & 13 \\
4 & 5
\end{array}\right) \\
& =\left(\begin{array}{cc}
95 & 109 \\
40 & 53
\end{array}\right) \\
& S U M(3, P S, P T)=S U M(2, P S, P T)+\left[\left(\begin{array}{ll}
7 & 3 \\
1 & 2
\end{array}\right)+\left(\begin{array}{ll}
8 & 6 \\
5 & 4
\end{array}\right)+\left(\begin{array}{ll}
9 & 9 \\
9 & 6
\end{array}\right)\right]\left(\begin{array}{ll}
6 & 7 \\
3 & 6
\end{array}\right) \\
& =\left(\begin{array}{c}
3+1 \\
4
\end{array}\right)\left(\begin{array}{cc}
15 & 27 \\
30 & 48
\end{array}\right)+\left(\begin{array}{c}
3+1 \\
3
\end{array}\right)\left(\begin{array}{cc}
44 & 70 \\
28 & 38
\end{array}\right)+\left(\begin{array}{c}
3+1 \\
2
\end{array}\right)\left(\begin{array}{cc}
17 & 13 \\
4 & 5
\end{array}\right) \\
& =\left(\begin{array}{ll}
293 & 385 \\
166 & 230
\end{array}\right)
\end{aligned}
$$

A product $=\left(K_{1}+E_{1}\right) \cdots\left(K_{M}+E_{M}\right)=\sum F_{1} \cdots F_{M}, F_{i}=E_{i}$ or $K_{i}$

Here's another extension: Let $F_{i}=E_{i}$ or $K_{i}$ or $R_{i}, R_{i}$ means $\left(K_{i}+E_{i}\right)$

So a product can be broken down into $2^{0}, 2^{1}, 2^{2}, \cdots, 2^{M}$ parts.

$S U M \_K\left(N, P S, P T, P F=F_{1} F_{2} \cdots F_{M}\right)=$ Sum of one part, $P F$ indicates the part.

Rewrite 3.1), add \{braces\}:

$$
\begin{aligned}
& \operatorname{SUM}(N, P S, P T)=\sum \prod_{i=1}^{M} A_{q}\left(\begin{array}{c}
A \\
M_{q}
\end{array}\right), A_{q}=\prod_{i=1}^{M} B_{i} \\
& B_{i}=\left\{\begin{array}{l}
\left\{\left(T_{i}-m\right) D_{i}\right\} ; X_{i}=T_{i}, m=\text { count of }\left\{\cdots, X_{i-1}\right\} \in K \\
\left\{K_{i}\right\}+\left\{m D_{i}\right\} ; F_{i} \neq R_{i}, X_{i}=K_{i}, m=\text { count of }\left\{\cdots, X_{i-1}\right\} \in T \\
\left\{K_{i}+m D_{i}\right\} ; F_{i}=R_{i}, X_{i}=K_{i}, m=\text { count of }\left\{\cdots, X_{i-1}\right\} \in T
\end{array}\right.
\end{aligned}
$$

Expand $S U M()$ by $\{$ braces $\}$ : 
3.2) $S U M_{-} K(N, P S, P T, P F)=\sum$ Expansion with same $\left\{K_{i}, R_{i}\right\} \in P F$

Use the similar method of [2] to prove.

\section{Coefficient Analysis}

Define $H_{1}(P S, P T, C)=A_{M-C}$ of 3.1), $C=$ Count of $X \in T, T \in$ Ring . It's the coefficient in the expression. Here $T \in$ Ring is just for analysis. $F_{M}^{K}=\sum M$ - product with different factors $\in K$, the sum traverse all combinations.

$E_{M}^{K}=\sum M$ - product with factors $\in K$, the sum traverse all combinations.

$F_{M}^{\{1,2, \cdots, N\}}$ is abbreviated as $F_{M}^{N}, E_{M}^{\{1,2, \cdots, N\}}$ is abbreviated as $E_{M}^{N}$;

By definition:

$$
\begin{aligned}
& E_{M}^{N}=\sum_{N_{1}+\cdots+N_{C}=M} 1^{N_{1}} 2^{N_{2}} \cdots N^{N_{C}} \\
& F_{0}^{K}=E_{0}^{K}=1 ; F_{M>|K|}^{K}=0 \\
& E_{M}^{N+1}=(N+1) E_{M-1}^{N+1}+E_{M}^{N} ; F_{M}^{\left[K, K_{M+1}\right]}=K_{M+1} F_{M-1}^{K}+F_{N}^{K} \\
& \text { 4.1) } H_{1}(K, T, 0)=\prod K_{i}, H_{1}(K, T, M)=\prod T_{i} \prod D_{i}
\end{aligned}
$$

[4] has proved:

$$
\text { 4.2) } \begin{aligned}
& H_{1}\left(\left[D \times A: D, K_{1}: D_{1}, \cdots, K_{M}: D_{M}\right],\left[A, T_{1}, \cdots, T_{M}\right], C\right) \\
= & D \times A \times H_{1}\left(\left[K_{1}: D_{1}, \cdots, K_{M}: D_{M}\right],\left[T_{1}, \cdots, T_{M}\right], C\right) \\
& +D \times A \times H_{1}\left(\left[K_{1}: D_{1}, \cdots, K_{M}: D_{M}\right],\left[T_{1}, \cdots, T_{M}\right], C-1\right)
\end{aligned}
$$

this $\rightarrow$

4.3) $\operatorname{SUM}\left(N,\left[1,2, \cdots, n, K_{1}: D_{1}, \cdots, K_{M}: D_{M}\right],\left[1,2, \cdots, n, T_{1}, \cdots, T_{M}\right]\right)$, $P T=\left[1, \cdots, n, T_{1}, \cdots, T_{M}\right]$ can use the form: $\left(T_{1}+K_{1}\right) \cdots\left(T_{M}+K_{M}\right)=n ! \sum A_{q}\left(\begin{array}{c}N+P B(P T)+n \\ I D X(P T)-q\end{array}\right)$

[2] has proved:

4.4) $H_{1}\left(\left[D \times T_{1}: D, \cdots, D \times T_{M}: D\right],\left[T_{1}, \cdots, T_{M}\right], C\right)=D^{M}\left(\begin{array}{c}M \\ C\end{array}\right) \prod T_{i}$ $D=1$, this $\rightarrow$

4.5) $\operatorname{SUM}(N, P T, P T)=M I N(P T)\left(\begin{array}{c}N+P B(P T)+P M(P T) \\ I D X(P T)\end{array}\right)$

This is a generalization of $\sum_{n=0}^{N}\left(\begin{array}{c}n \\ M\end{array}\right)=\left(\begin{array}{c}N+1 \\ M+1\end{array}\right)$

Eg: $\operatorname{SUM}(2,[1,2,4])=1 \times 2 \times 4+1 \times 2 \times 5+2 \times 3 \times 5=1 \times 2 \times 4\left(\begin{array}{c}2+1+3 \\ 5\end{array}\right)$

4.6) $H_{1}(P S, P T, C)=\nabla^{C} S U M(C, P S, P T)$, $\nabla^{I D X(P T)} \operatorname{SUM}(N>M, \cdots)=\prod T_{i} \prod D_{i}$

The last row value of the difference sequence is not arbitrary.

Comparison with 4.4), [4] has proved:

4.7) if $T_{i}+1=T_{i+1}$, then 


$$
\begin{aligned}
& \left(K_{i}+1=K_{i+1}, H_{1}\left(\left[D \times K_{1}: D, \cdots\right], P T, C\right)=D^{M}\left(\begin{array}{c}
M \\
C
\end{array}\right) T_{1} \cdots T_{C} K_{C+1} \cdots K_{M}\right. \\
& K_{i}-1=K_{i+1}, H_{1}\left(\left[D \times K_{1}: D, \cdots\right], P T, C\right)=D^{M}\left(\begin{array}{c}
M \\
C
\end{array}\right) T_{1} \cdots T_{C} K_{1} \cdots K_{M-C} \\
& K_{i}+1=K_{i+1}, H_{1}\left(\left[D \times K_{1}:-D, \cdots\right], P T, C\right)=(-1)^{C} D^{M}\left(\begin{array}{c}
M \\
C
\end{array}\right) T_{1} \cdots T_{C} K_{1} \cdots K_{M-C} \\
& K_{i}-1=K_{i+1}, H_{1}\left(\left[D \times K_{1}:-D, \cdots\right], P T, C\right)=(-1)^{C} D^{M}\left(\begin{array}{c}
M \\
C
\end{array}\right) T_{1} \cdots T_{C} K_{C+1} \cdots K_{M} \\
& {[x+y]_{n}=\nabla \operatorname{SUM}(y+1,[x-n+1, x-n+2, \cdots, x])} \\
& =\left(\begin{array}{l}
y \\
n
\end{array}\right)\left(\begin{array}{l}
n \\
n
\end{array}\right) n ![x]_{0}+\left(\begin{array}{c}
y \\
n-1
\end{array}\right)\left(\begin{array}{c}
n \\
n-1
\end{array}\right)(n-1) ![x]_{1}+\cdots+\left(\begin{array}{l}
y \\
0
\end{array}\right)\left(\begin{array}{l}
n \\
0
\end{array}\right) 0 ![x]_{n} \\
& =\left(\begin{array}{l}
n \\
n
\end{array}\right)[y]_{n}[x]_{0}+\left(\begin{array}{c}
n \\
n-1
\end{array}\right)[y]_{n-1}[x]_{1}+\cdots+\left(\begin{array}{l}
n \\
0
\end{array}\right)[y]_{0}[x]_{n}
\end{aligned}
$$

$\rightarrow$ Vandermonde identity [5]: $[x+y]_{n}=\sum_{k=0}^{n}\left(\begin{array}{l}n \\ k\end{array}\right)[x]_{k}[y]_{n-k}$

$$
\begin{aligned}
{[x+y]^{n} } & =\nabla \operatorname{SUM}(-y+1,[x:-1, x+1:-1, \cdots, x+n-1:-1]) \\
& =(-1)^{n}\left(\begin{array}{c}
-y \\
n
\end{array}\right)\left(\begin{array}{l}
n \\
n
\end{array}\right) n ![x]^{0}+(-1)^{n-1}\left(\begin{array}{c}
-y \\
n-1
\end{array}\right)\left(\begin{array}{c}
n \\
n-1
\end{array}\right)(n-1) ![x]^{1}+\cdots \\
& =\left(\begin{array}{c}
n \\
n
\end{array}\right)[y]^{n}[x]^{0}+\left(\begin{array}{c}
n \\
n-1
\end{array}\right)[y]^{n-1}[x]^{1}+\cdots+\left(\begin{array}{c}
n \\
0
\end{array}\right)[y]^{0}[x]^{n}
\end{aligned}
$$

$\rightarrow$ Norlund identity [5]: $[x+y]^{n}=\sum_{k=0}^{n}\left(\begin{array}{l}n \\ k\end{array}\right)[x]^{k}[y]^{n-k}$

$$
\begin{aligned}
& \left(K_{1}+N \times D_{1}\right) \cdots\left(K_{M}+N \times D_{M}\right) \\
& =\operatorname{VSUM}\left(N+1,\left[K_{1}: D_{1} . . K_{M}: D_{M}\right]\right)=\sum_{q=0}^{M} A_{q}\left(\begin{array}{c}
N \\
q
\end{array}\right)
\end{aligned}
$$

4.8) $\left(K_{1}+N \times D_{1}\right) \cdots\left(K_{M}+N \times D_{M}\right)$ can be decomposed to $\sum_{q=0}^{M} C_{q}\left(\begin{array}{l}N \\ q\end{array}\right)$ by 3.1)

$$
\begin{aligned}
& N=x, \quad K_{i}=i-1, \quad P S=[0,1,2, \cdots, M-1] \rightarrow \\
& {[x]^{M}=\left(\begin{array}{c}
x \\
M
\end{array}\right)\left(\begin{array}{l}
M \\
M
\end{array}\right) M ![M-1]_{0}} \\
& +\left(\begin{array}{c}
x \\
M-1
\end{array}\right)\left(\begin{array}{c}
M \\
M-1
\end{array}\right)(M-1) ![M-1]_{1} \cdots\left(\begin{array}{c}
x \\
0
\end{array}\right)\left(\begin{array}{c}
M \\
0
\end{array}\right) 0 ![M-1]_{M} \\
& \text { 4.9) }[x]^{M}=\sum_{k=0}^{M}\left(\begin{array}{c}
M \\
k
\end{array}\right)[x]_{k}[M-1]_{M-k} \\
& N=x, \quad K_{i}=1-i, \quad P S=[0:-1,-1:-1, \cdots,-M+1:-1] \rightarrow \\
& {[-x]_{M}=(-1)^{M+0}\left(\begin{array}{l}
X \\
M
\end{array}\right)\left(\begin{array}{l}
M \\
M
\end{array}\right) M ![M-1]_{0}} \\
& +(-1)^{(M-1)+1}\left(\begin{array}{c}
X \\
M-1
\end{array}\right)\left(\begin{array}{c}
M \\
M-1
\end{array}\right)(M-1) ![M-1]_{1}
\end{aligned}
$$


4.10) $[-x]_{M}=\sum_{k=0}^{M}(-1)^{M}\left(\begin{array}{c}M \\ k\end{array}\right)[x]_{k}[M-1]_{M-K}$

[4] has proved:

4.11) if $T_{i}+1=T_{i+1}$, then

$H_{1}\left(\left[D \times K_{1}: D, \cdots, D \times K_{M}: D\right],\left[T_{1}, \cdots, T_{M}\right], C\right)$

$=D^{C} T_{1} \cdots T_{C}\left[F_{M-C}^{D \times K} E_{0}^{D \times\{1,2, \cdots, C\}}+F_{M-C-1}^{D \times K} E_{1}^{D \times\{1,2, \cdots, C\}}+\cdots+F_{0}^{D \times K} E_{M-C}^{D \times\{1,2, \cdots, C\}}\right]$

This $\rightarrow K_{i}$ can exchange order in $\operatorname{SUM}\left(N,\left[K_{1}, \cdots, K_{M}\right]\right)$.

In fact, $K_{i}$ can exchange order in $\operatorname{SUM}\left(N,\left[K_{1}: D_{1}, \cdots, K_{M}: D_{M}\right]\right)$ by definition.

This $\rightarrow$

4.12) if $\lambda$ is a primitive unit root, $\lambda^{M}=1$, then

$\operatorname{SUM}\left(N,\left[\lambda^{1}, \lambda^{2}, \cdots, \lambda^{M}\right]\right)$

$=M !\left(\begin{array}{c}N \\ M+1\end{array}\right) E_{0}^{M}+(M-1) !\left(\begin{array}{c}N \\ M\end{array}\right) E_{1}^{M-1}+\cdots$

$+1 !\left(\begin{array}{c}N \\ 2\end{array}\right) E_{M-1}^{1}+0 !\left(\begin{array}{c}N \\ 1\end{array}\right)(-1)^{M+1}$

$\left(\lambda^{1}+1\right) \cdots\left(\lambda^{M}+1\right)=\operatorname{SUM}(2,[\cdots])-\operatorname{SUM}(1,[\cdots])=1+(-1)^{M+1}$

It's obvious when $M$ is even; if $M$ is odd then $\left(\lambda^{1}+1\right) \cdots\left(\lambda^{M-1}+1\right)=1$

$\left(\lambda^{1}+2\right) \cdots\left(\lambda^{M}+2\right)=\operatorname{SUM}(3,[\cdots])-\operatorname{SUM}(2,[\cdots])=2^{M}+(-1)^{M+1}$

It can be concluded from the definition:

4.13) 1) $H_{1}([1, \cdots, M],[1, \cdots, 2 M-1], C)$

$=\sum_{P M()=M, P B()=C} M I N(P S)+\sum_{P M()=M, P B()=C-1} M I N(P S)$

2) $H_{1}([2, \cdots, M],[3, \cdots, 2 M-1], C)=\sum_{P M(P S)=M, P B(P S)=C} M I N(P S)$

$P S$ are Basic Shapes.

\section{Special Functions}

5.1) $\operatorname{SUM}(N,[a: 0])=a\left(\begin{array}{c}N \\ 1\end{array}\right)$

5.2) $\operatorname{SUM}(N,[a: d])=\sum_{n=0}^{N-1}(a+n d)=d\left(\begin{array}{l}N \\ 2\end{array}\right)+a\left(\begin{array}{c}N \\ 1\end{array}\right)$

5.3) $\operatorname{SUM}(N,[1,2, \cdots, M])=M !\left(\begin{array}{c}N+M \\ M+1\end{array}\right)$

5.4) $\operatorname{SUM}(N,[1,2, \cdots, M],[1,3, \cdots, 2 M-1])=\sum I_{1} I_{2} \cdots I_{M}$, $1 \leq I_{1}<I_{2}<\cdots<I_{M} \leq N+M-1$

5.5) $\operatorname{SUM}(N,[1,1, \cdots, 1],[1,2, \cdots, M])=1^{M}+2^{M}+\cdots+N^{M}$

5.6) $\operatorname{SUM}\left(N,\left[0: D_{1}, \cdots, 0: D_{M}\right], P T\right)=S U M_{-} K\left(N, P S, P T, E_{1} \cdots E_{M}\right)$

5.7) $\operatorname{SUM}(N,[1,1, \cdots, 1],[1,3, \cdots, 2 M-1])=E_{M}^{N}$ 


\section{Stirling, Lah Number}

$S_{1}(M, K), S_{2}(M, K)$ is unsigned Stirling number. $L_{M, K}$ is Lah number.

[1] use 4.5) to calculate

$S_{1}(N, N-M)=F_{M}^{N-1}=\sum M I N(P S)\left(\begin{array}{c}N+P B(P T)+P M(P T) \\ I D X(P T)\end{array}\right), P S$ traverses all

Basic Shapes, $P M(P S)=M$

This conforms to 4.13). In this paper, it can be written as:

6.1) $\operatorname{SUM}(N,[1,2, \cdots, M],[1,3, \cdots, 2 M-1])=S_{1}(N+M, N)$, this is 5.4)

$E_{M}^{N} \stackrel{\text { def }}{\longrightarrow} \sum_{N_{1}+\cdots+N_{C}=M} 1^{N_{1}} 2^{N_{2}} \cdots N^{N_{C}}$, It's a known property of $S_{2}(N+M, N)$

6.2) $\operatorname{SUM}(N,[1, \cdots, 1],[1,3, \cdots, 2 M-1])=E_{M}^{N}=S_{2}(N+M, N)$

6.3) 1) $H_{1}([1, \cdots, 1],[1, \cdots, M], K)$

$$
=K ! S_{2}(M+1, K+1)=K ! \sum_{i=0}^{M-K}\left(\begin{array}{c}
M \\
i
\end{array}\right) S_{2}(M-i, K)
$$

2) $H_{1}([1, \cdots, 1],[2, \cdots, M], K)=(K+1) ! S_{2}(M, K+1)$

[Proof]

Definition of $S_{2}(M, K)$ is

$$
\begin{aligned}
& N^{M}=\sum_{K=0}^{M} S_{2}(M, K)[N]_{k}=\sum_{K=0}^{M} K ! S_{2}(M, K)\left(\begin{array}{l}
N \\
K
\end{array}\right) \\
& (N+1)^{M}=\nabla \operatorname{SUM}(N+1,[1, \cdots, 1],[1, \cdots, M]) \\
& =\sum_{q=0}^{M} A_{q}\left(\begin{array}{c}
N \\
q
\end{array}\right)=\sum_{K=0}^{M} K ! S_{2}(M, K)\left(\begin{array}{c}
N+1 \\
K
\end{array}\right) \\
& =\sum_{K=0}^{M} K ! S_{2}(M, K)\left(\begin{array}{c}
N \\
K+1
\end{array}\right)+\sum_{K=0}^{M} K ! S_{2}(M, K)\left(\begin{array}{c}
N \\
K
\end{array}\right) \\
& H_{1}([1, \cdots, 1],[1, \cdots, M], K)=K ! S_{2}(M, K)+(K+1) ! S_{2}(M, K+1) \rightarrow \text { the first }
\end{aligned}
$$
equation

$\stackrel{4.11)}{\longrightarrow} H_{1}(\cdots, K)=K !\left[F_{M-K}^{[1, \cdots, 1]} E_{0}^{K}+\cdots+F_{0}^{[1, \cdots, 1]} E_{M-K}^{K}\right] \stackrel{F_{M-K}^{[1, \cdots, 1]}=\left(\begin{array}{c}M \\ M-K\end{array}\right)}{\longrightarrow}$ the second equation

$$
\begin{aligned}
& \stackrel{4.2)}{\longrightarrow} H_{1}([1, \cdots, 1],[1, \cdots, M], K) \\
& =H_{1}([1, \cdots, 1],[2, \cdots, M], K)+H_{1}([2, \cdots, M], K-1) \\
& \text { q.e.d. }
\end{aligned}
$$

This $\rightarrow S_{2}(N+1, M)=\sum_{k=M-1}^{N}\left(\begin{array}{l}N \\ k\end{array}\right) S_{2}(K, M-1)$, which is recorded in [5]

Example 6.1:

Directly according to the definition of $H_{1}()$

$$
\begin{aligned}
& H_{1}(\cdots, 1)=(1 \times 2 \times 2 \times \cdots \times 2+1 \times 1 \times 2 \times \cdots \times 2+\cdots+1 \times 1 \times \cdots \times 1 \times 1) \\
& \rightarrow S_{2}(M+1,2)=2^{M}-1
\end{aligned}
$$




$$
\begin{aligned}
& H_{1}(\cdots, M-1)=(M-1) !(1+2+\cdots+M) \rightarrow S_{2}(M+1, M)=\left(\begin{array}{c}
M+1 \\
2
\end{array}\right) \\
& \text { 6.4) }\left(\begin{array}{c}
M \\
K
\end{array}\right) \frac{M !}{K !}=S_{1}(M+1, K+1) S_{2}(K, K)+\cdots+S_{1}(M+1, M+1) S_{2}(M, K)
\end{aligned}
$$

[Proof]

$$
\begin{aligned}
& H_{1}([1, \cdots, M],[1, \cdots, M], K) \stackrel{4.4)}{\longrightarrow}=\left(\begin{array}{c}
M \\
K
\end{array}\right) M ! \\
& \stackrel{4.11)}{\longrightarrow}=K !\left[F_{M-K}^{M} E_{0}^{K}+\cdots+F_{0}^{M} E_{M-K}^{K}\right] \\
& =K !\left[S_{1}(M+1, K+1) S_{2}(K, K)+\cdots+S_{1}(M+1, M+1) S_{2}(M, K)\right]
\end{aligned}
$$

q.e.d.

Definition of Lah number [5] is $[-X]_{M}=\sum_{k=0}^{M} L_{M, K}[x]_{k} \stackrel{4.10)}{\longrightarrow}$

6.5) $L_{M, K}=(-1)^{M} \frac{M !}{K !}\left(\begin{array}{c}M-1 \\ K-1\end{array}\right)$, this is recorded in [5].

\section{Congruence Analysis}

$P$ is a prime number, we already know:

(7*) $S_{1}(P, P-K)=F_{K}^{P-1} \equiv 0 M O D P, 0<K<P-1$

[1] has proved, it is easy to infer from 4.5):

7.1) $S U M(P-P B(P T)-P M(P T), P T, P T)$

$$
=\operatorname{MIN}(P T)\left(\begin{array}{c}
P \\
I D X(P T)
\end{array}\right) \equiv 0 M O D P, I D X(P T)<P
$$

E.g.: $1 \times 2+2 \times 3+3 \times 4 \equiv 1 \times 3+1 \times 4+2 \times 4 \equiv 0 M O D 5$

This is the promotion of $\left(7^{*}\right)$.

[4] has proved, it is easy to infer from 3.1):

7.2) For arbitrary $K_{i} \in Z$

1) $M<P-1, \operatorname{SUM}\left(P,\left[K_{1}: D, \cdots, K_{M}: D\right]\right) \equiv 0 M O D P$

2) $M=P-1,(D, P)=1, \operatorname{SUM}\left(P,\left[K_{1}: D, \cdots, K_{M}: D\right]\right) \equiv-1 M O D P$

$\operatorname{SUM}\left(P,\left[K_{1}, \cdots, K_{M}\right]\right)=\prod K_{i}+\prod\left(1+K_{i}\right)+\cdots+\prod\left(P-1+K_{i}\right)$. Exclude products $\equiv 0 M O D P$ :

Example 7.1:

$$
\begin{aligned}
& 1^{Q}+2^{Q}+\cdots+(P-1)^{Q} \equiv 0 M O D P, Q<P-1 ; \equiv-1 M O D P, Q=P-1 \\
& 1^{2} \times 2 \times 3+2^{2} \times 3 \times 4+\cdots+(P-3)^{2} \times(P-2) \times(P-1) \equiv-1 M O D P, \quad P=5 ; \\
& \equiv 0 M O D P, \quad P>5 \\
& 1^{3} \times 2+2^{3} \times 3+\cdots+(P-2)^{3} \times(P-1) \equiv-1 M O D P, P=5 ; \equiv 0 M O D P, P>5 \\
& 1 \times 2^{3}+2 \times 3^{3}+\cdots+(P-2) \times(P-1)^{3} \equiv-1 M O D P, \quad P=5 ; \equiv 0 M O D P, P>5 \\
& 1^{2} \times 2^{2}+2^{2} \times 3^{2}+\cdots+(P-2)^{2} \times(P-1)^{2} \equiv-1 M O D P, \quad P=5 ; \equiv 0 M O D P, P>5
\end{aligned}
$$

In 1), let $P S=\left[K_{1}=1, \cdots, K_{M}\right]$ is a Basic Shape and $K_{M}=P-1$

Rewrite $P S=\llbracket L_{1} L_{2} \cdots L_{Q} \rrbracket, L_{i}=$ count of continuity. $\left(L_{i}, L_{i+1}\right)$ means a 
discontinuity.

$\llbracket L_{1} \cdots L_{Q} \rrbracket$ can been slided to $\left[L_{Q}, L_{1}, L_{2}, \cdots\right],\left[L_{Q-1}, L_{Q}, L_{1}, L_{2}, \cdots\right]$ by $S U M(P, P S) M O D P$

[3] has proved:

7.3) $\sum M I N(P S) \equiv 0 M O D P$

$\{P S\}=$ All of the Basic Shapes $\left[1, \cdots, K_{M}=P-1\right] \neq[1,2, \cdots, P-1]$ can slide to.

Example 7.2:

$$
\begin{aligned}
& 1 \times(3 \times 4 \times 5) \times(7 \times 8) \times 10+1 \times 3 \times(5 \times 6 \times 7) \times(9 \times 10) \\
& +(1 \times 2) \times 4 \times 6 \times(8 \times 9 \times 10)+(1 \times 2 \times 3) \times(5 \times 6) \times 8 \times 10 \\
& =139260 \equiv 0 M O D 11
\end{aligned}
$$

This $\rightarrow$ [1] has proved:

7.4) $\sum M I N(P S) \equiv 0 M O D P,\{P S\}=$ All of the Basic Shapes with the same $P M()$ and the same $P B(), P B()>0$ and $K_{M}=P-1$

In Example 7.1, the $S U M()$ is not symmetrical, it's part of some symmetrical express.

E.g.: $\left(1^{2} \times 2^{2}+2^{2} \times 3^{2}+3^{3} \times 4^{2}\right)+\left(1^{2} \times 3^{2}+2^{2} \times 4^{2}+4^{2} \times 1^{2}\right)$

$$
\equiv \operatorname{SUM}(5,[1,1,2,2])+\operatorname{SUM}(5,[1,1,3,3]) M O D 5
$$

Use $F(P S)=$ The symmetrical express. Obviously: $F\left(\left[K_{1} \cdots K_{M}\right]\right) \equiv 0 M O D P$, $M<P-1$;

7.5) $F(\lambda(P S)) \equiv-\frac{\left(\begin{array}{c}P-1 \\ L E N(P S)\end{array}\right) \frac{\left(\mu_{1}+\mu_{2}+\cdots\right) !}{\mu_{1} ! \mu_{2} ! \cdots}}{P-L E N(P S)} M O D P, M=P-1$

[Proof]

Use $C N T(P S)=$ Count of $P S \in F(P S) \rightarrow F(P S) \equiv-C N T(P S) M O D P$

Use $L E N(P S)=$ Count of different $K_{i} \in P S$

Obviously:

$\operatorname{LEN}(P S \in F(P S))$ is same, count of products $\in F(P S)=P-L E N(P S)$

$C N T(P S)=[$ Count of products $\in F(P S)] /(P-L E N(P S))$

Use $\lambda(P S)$ to classify $P S$.

$$
\begin{aligned}
& \lambda(P S):= 1^{\lambda_{1}} 2^{\lambda_{2}} \cdots(P-1)^{\lambda_{p-1}}, P M(P S)=1 \times \lambda_{1}+2 \times \lambda_{2}+\cdots+(P-1) \times \lambda_{p-1} \\
& \lambda(P S):=4^{1}: 1^{4}+2^{4}+3^{4}+\cdots \\
& \lambda(P S):=1^{4}: 1 \times 2 \times 3 \times 4+\cdots \\
& \lambda(P S):=1^{1} 3^{1}: 1^{3} \times 2+2^{3} \times 3+\cdots ; 1^{3} \times 3+2^{3} \times 4+\cdots ; \quad 1 \times 2^{3}+2 \times 3^{3}+\cdots ; 1 \times 3^{3}+2 \times 4^{3}+\cdots \\
& \lambda(P S):=2^{2}: 1^{2} \times 2^{2}+2^{2} \times 3^{2}+\cdots ; 1^{2} \times 3^{2}+2^{2} \times 4^{2}+\cdots ; 1^{2} \times 4^{2}+2^{2} \times 5^{2}+\cdots \\
& \lambda(P S):=1^{2} 2^{1}: 1^{2} \times 2 \times 3+\cdots ; 1 \times 2^{2} \times 3+\cdots ; 1 \times 2 \times 3^{2}+\cdots ; 1^{2} \times 3 \times 5+\cdots
\end{aligned}
$$


1) The combination number of $L E N(P S)$ in $P-1=\left(\begin{array}{c}P-1 \\ L E N(P S)\end{array}\right)$

2) Record $\lambda(P S)$ as $\left\{\mu_{1}, \mu_{2}, \cdots\right\}$

There are $\mu_{1}$ numbers in $\lambda_{1}, \lambda_{2}, \cdots$ equal to $x>0$

There are $\mu_{2}$ numbers in $\lambda_{1}, \lambda_{2}, \cdots$ equal to $y>0, y \neq x$

The combination number of $\mu_{1}, \mu_{2}, \cdots=\frac{\left(\mu_{1}+\mu_{2}+\cdots\right) !}{\mu_{1} ! \mu_{2} ! \cdots}$

Count of products $\in F(P S)=\left(\begin{array}{c}P-1 \\ L E N(P S)\end{array}\right) \frac{\left(\mu_{1}+\mu_{2}+\cdots\right) !}{\mu_{1} ! \mu_{2} ! \cdots}$

q.e.d.

Example 7.3:

$\left(1^{3} \times 2+2^{3} \times 3+3^{3} \times 4\right)+\left(1^{3} \times 3+2^{3} \times 4+4^{3} \times 1\right)+\left(1^{3} \times 4+3^{3} \times 1+4^{3} \times 2\right)$

$+\left(1 \times 2^{3}+2 \times 3^{3}+3 \times 4^{3}\right) \equiv-\frac{4 !}{2 ! 2 !} \times \frac{2 !}{1 ! 1 !} /(5-2) \equiv-4 M O D P$

$\left(1^{2} \times 2^{2}+2^{2} \times 3^{2}+3^{2} \times 4^{2}\right)+\left(1^{2} \times 3^{2}+2^{2} \times 4^{2}+4^{2} \times 1^{2}\right)$

$\equiv-\frac{4 !}{2 ! 2 !} \times \frac{2 !}{2 !} /(5-2) \equiv-2 M O D P$

$\left(1^{2} \times 2 \times 3+2^{2} \times 3 \times 4\right)+\left(1^{2} \times 2 \times 4+3^{2} \times 4 \times 1\right)+\left(1^{2} \times 3 \times 4+4^{2} \times 1 \times 2\right)$

$+\left(1 \times 2^{2} \times 3+2 \times 3^{2} \times 4\right)+\left(1 \times 2^{2} \times 4+3 \times 4^{2} \times 1\right)+\left(1 \times 2 \times 3^{2}+2 \times 3 \times 4^{2}\right)$

$\equiv-\frac{4 !}{3 ! 1 !} \times \frac{3 !}{2 ! 1 !} /(5-3) \equiv-6$ MOD P

7.6) 1) $K ! S_{2}(P-1, K)=K ! E_{P-1-K}^{K} \equiv(-1)^{K+1} M O D P, 1 \leq K \leq P-1$

2) $S_{2}(P, K) \equiv 0 M O D P, 1<K<P$

[Proof]

$\operatorname{SUM}(N,[1,2, \cdots, P-1]) \stackrel{4.11)\left(7^{*}\right)}{\longrightarrow}$

$\equiv(P-1) !\left(\begin{array}{c}N \\ P\end{array}\right) E_{0}^{P-1}+\cdots+1 !\left(\begin{array}{c}N \\ 2\end{array}\right) E_{P-2}^{1}+0 !\left(\begin{array}{c}N \\ 1\end{array}\right)(P-1) ! M O D P$

$\operatorname{\nabla SUM}(N,[1, \cdots, P-1])$

$\equiv(P-1) !\left(\begin{array}{l}N-1 \\ P-1\end{array}\right) E_{0}^{P-1}+\cdots+1 !\left(\begin{array}{c}N-1 \\ 1\end{array}\right) E_{P-2}^{1}+0 !\left(\begin{array}{c}N-1 \\ 0\end{array}\right)(p-1) ! M O D P$

$[P]_{P-1}=\operatorname{\nabla SUM}(2,[1, \cdots, P-1]) \equiv 1+(P-1) ! \equiv 0 M O D P$

$\rightarrow(P-1) ! \equiv-1 M O D P$

This is a new proof of Wilson theorem.

$1 ! E_{P-2}^{1}=1 \equiv 1 M O D P$

$[P+1]_{P-1}=\nabla \operatorname{SUM}(3,[1, \cdots, P-1]) \equiv 0 M O D P \rightarrow 2 ! E_{P-3}^{2} \equiv-1 M O D P$

$[P+2]_{P-1}=\nabla \operatorname{SUM}(4,[1, \cdots, P-1]) \equiv 0 M O D P \rightarrow 3 ! E_{P-4}^{3} \equiv 1 M O D P$ 


$$
\begin{aligned}
& \rightarrow 1) \\
& S_{2}(P, K)=S_{2}(P-1, K-1)+K \times S_{2}(P-1, K) \rightarrow \\
& \left.(K-1) ! S_{2}(P, K)=(K-1) ! S_{2}(P-1, K-1)+K ! S_{2}(P-1, K) \rightarrow 2\right) \\
& \equiv(-1)^{K}+(-1)^{K+1} \equiv 0 \text { MOD } P
\end{aligned}
$$

q.e.d.

$N^{P-1} \equiv 1 M O D P=\sum_{K=0}^{P-1} K ! S_{2}(P-1, K)\left(\begin{array}{l}N \\ K\end{array}\right) \stackrel{7.6), S_{2}(P-1,0)=0}{\longrightarrow}$

7.7) $-\left(\begin{array}{c}N \\ P-1\end{array}\right)+\left(\begin{array}{c}N \\ P-2\end{array}\right)+\cdots-\left(\begin{array}{c}N \\ 2\end{array}\right)+\left(\begin{array}{c}N \\ 1\end{array}\right) \equiv 1 M O D P,(N, P)=1$

7.8) $\sum_{n=1}^{P-1} n^{P-2} \equiv 0 M O D P^{2}, P>3$

[Proof]

$$
\begin{aligned}
& P^{P-2}+\sum_{n=1}^{P-1} n^{P-2}=\sum_{n=1}^{P} n^{P-2}=\operatorname{SUM}(P,[1, \cdots, 1],[1, \cdots, P-2]) \\
& \stackrel{6.3)}{\longrightarrow}=\sum_{k=0}^{P-2} k ! S_{2}(P-1, k+1)\left(\begin{array}{c}
P \\
K+1
\end{array}\right) \\
& =\sum_{k=1}^{\frac{P-1}{2}}\left[(k-1) ! S_{2}(P-1, k)\left(\begin{array}{l}
P \\
k
\end{array}\right)+(P-k-1) ! S_{2}(P-1, P-k)\left(\begin{array}{c}
P \\
P-k
\end{array}\right)\right] \\
& \stackrel{7.6)}{\longrightarrow} \equiv \sum_{k=1}^{\frac{P-1}{2}}\left[(-1)^{K+1}+(-1)^{P-K+1}\right]\left(\begin{array}{l}
P \\
K
\end{array}\right) \\
& \equiv \sum_{k=1}^{\frac{P-1}{2}} P \times \lambda_{k}\left(\begin{array}{l}
P \\
K
\end{array}\right) M O D P \equiv 0 \text { MOD } P^{2}
\end{aligned}
$$

q.e.d.

\section{Form 2 and Analysis}

Rewrite (1) of section 3 as

$\sum_{n=0}^{N-1} n\left(\begin{array}{c}n+K \\ M\end{array}\right)=(M+1)\left(\begin{array}{c}N+K+1 \\ M+2\end{array}\right)-(1+K)\left(\begin{array}{c}N+K \\ M+1\end{array}\right)$

$P S=\left[K_{1}: D_{1}, \cdots, K_{M}: D_{M}\right], P T=\left[T_{1}, \cdots, T_{M}\right]$, use the same method of 3.1) to prove:

8.1) Use the Form $_{2}=\left(T_{1}+K_{1}\right) \cdots\left(T_{M}+K_{M}\right)=\sum X_{1} \cdots X_{M}$, $\operatorname{SUM}(N, P S, P T)=\sum A_{q}\left(\begin{array}{c}N+T_{M}-q \\ I D X(P T)-q\end{array}\right), q=$ count of $X \in K . A_{q}=\prod_{i=1}^{M} B_{i}$ $B_{i}=\left\{\begin{array}{l}\left(T_{i}-m\right) D_{i} ; X_{i}=T_{i}, m=\text { count of }\left\{\cdots, X_{i-1}\right\} \in K \\ K_{i}+\left(m-T_{i}\right) D_{i} ; X_{i}=K_{i}, m=\text { count of }\left\{\cdots, X_{i-1}\right\} \in K\end{array}\right.$

Define $H_{2}(P S, P T, C)=A_{M-q}$ of 8.1), $C=$ Count of $X \in T$

8.2) $H_{2}(P T, P T, q<P M(P T))=0 ; H_{2}(P T, P T, P M(P T))=\prod T_{i}$, This $\rightarrow$ 4.5)

$$
\text { 8.3) } \begin{aligned}
& H_{2}([1, \cdots, M],[1, \cdots, 2 M-1], C) \\
& =(-1)^{M-C-1} H_{1}([2, \cdots, M],[3, \cdots, 2 M-1], C-1)
\end{aligned}
$$


[Proof]

$$
\begin{aligned}
& H_{2}([1, \cdots, 1],[1, \cdots, 2 M-1], C)=\prod_{i=1}^{M} B_{i}, \\
& B_{i}=\left\{\begin{array}{l}
\left(T_{i}-m\right) ; X_{i}=T_{i}, m=\text { count of }\left\{\cdots, X_{i-1}\right\} \in K \\
1+(m-2 i+1) ; X_{i}=K_{i}, m=\text { count of }\left\{\cdots, X_{i-1}\right\} \in K^{\stackrel{m=i-1-q}{\longrightarrow}}
\end{array}\right. \\
& =\left\{\begin{array}{l}
\left(T_{i}-m\right) ; X_{i}=T_{i}, m=\text { count of }\left\{\cdots, X_{i-1}\right\} \in K \\
-((i-1)+q) ; X_{i}=K_{i}, q=\text { count of }\left\{\cdots, X_{i-1}\right\} \in T
\end{array}\right. \\
& \rightarrow(-1)^{M-C} H_{2}(\cdots, C)+(-1)^{M-C-1} H_{2}(\cdots, C+1) \\
& =H_{1}([1, \cdots, M],[1, \cdots, 2 M-1], C) \stackrel{4.2)}{\longrightarrow} \\
& =H_{1}([2, \cdots, M],[3, \cdots, 2 M-1], C)+H_{1}([2, \cdots, M],[3, \cdots, 2 M-1], C-1) \\
& \rightarrow H_{2}([1, \cdots, M],[1, \cdots, 2 M-1], C) \\
& =(-1)^{M-C-1} H_{1}([2, \cdots, M],[3, \cdots, 2 M-1], C-1)
\end{aligned}
$$

[6] obtains the unified expression of $S_{1}(N, N-M)$ and $S_{2}(N, N-M)$ by induction:

$$
\begin{aligned}
& \text { 1) } S_{1}(N, N-M)=\sum_{k=1}^{M} A(M, k)\left(\begin{array}{c}
N \\
2 M-K+1
\end{array}\right) \\
& \text { 2) } S_{2}(N, N-M)=\sum_{k=1}^{M}(-1)^{k-1} A(M, k)\left(\begin{array}{l}
N+M-K \\
2 M-K+1
\end{array}\right)
\end{aligned}
$$

[Proof]

$$
\begin{aligned}
& S_{1}(N, N-M)=S U M(N-M,[1, \cdots, M],[1, \cdots, 2 M-1]), \\
& \text { Form } \left._{1}=\left(T_{2}+K_{2}\right) \cdots\left(T_{M}+K_{M}\right), 4.3\right) \rightarrow \\
& =\sum_{q=0}^{M-1} H_{1}([2, \cdots, M],[3, \cdots, 2 M-1], q)\left(\begin{array}{c}
N-M+(M-1)+1 \\
2 M-(M-q)
\end{array}\right) \stackrel{k=M-q}{\longrightarrow} \\
& \left.=\sum_{k=1}^{M} H_{1}([2, \cdots, M],[3, \cdots, 2 M-1], M-k)\left(\begin{array}{c}
N \\
2 M-k
\end{array}\right) \rightarrow 1\right) \\
& H_{1}([2, \cdots, M],[3, \cdots, 2 M-1], M-K)=A(M, K) \\
& S_{2}(N, N-M)=S U M(N-M,[1, \cdots, 1],[1, \cdots, 2 M-1]), \text { use the Form } 2 \\
& =\sum_{q=0}^{M} H_{2}([1, \cdots, 1],[1, \cdots, 2 M-1], q)\left(\begin{array}{c}
N-M+(2 M-1)-(M-q) \\
2 M-(M-q)
\end{array}\right) \\
& =\sum_{q=0}^{M} H_{2}([1, \cdots, 1],[1, \cdots, 2 M-1], q)\left(\begin{array}{c}
N-1+q \\
M+q
\end{array}\right) \stackrel{H_{2}(\cdots, 0)=0}{\longrightarrow} \\
& =\sum_{q=1}^{M} H_{2}([1, \cdots, 1],[1, \cdots, 2 M-1], q)\left(\begin{array}{c}
N-1+q \\
M+q
\end{array}\right) \stackrel{K=M-q+1}{\longrightarrow} \\
& =\sum_{K=1}^{M} H_{2}([1, \cdots, 1],[1, \cdots, 2 M-1], M-k+1)\left(\begin{array}{c}
N+M-K \\
2 M-K+1
\end{array}\right) \stackrel{8.3)}{\longrightarrow} \\
& \left.=\sum_{k=1}^{M}(-1)^{k-1} H_{1}([2, \cdots, M],[3, \cdots, 2 M-1], M-k)\left(\begin{array}{c}
N+M-k \\
2 M-k+1
\end{array}\right) \rightarrow 2\right)
\end{aligned}
$$

q.e.d. 
8.4) 1) $\sum_{C=0}^{C=M-1}(-1)^{M-1-C} \sum_{P M(P S)=M, P B(P S)=C} M I N(P S)=1$

2) $\sum_{C=0}^{C=M-1}(-1)^{M-1-C} \sum_{P M(P S)=M, P B(P S)=C}(M+2+C) M I N(P S)=2^{M+1}-1$

PS are Basic Shapes

[Proof]

$S_{2}(M+1,1)=1$, this is a known property $=\operatorname{SUM}(1,[1, \cdots, 1],[1, \cdots, 2 M-1])$ $\stackrel{\text { Use } \text { Form }_{3}}{\longrightarrow}$

$=\sum A_{q}\left(\begin{array}{c}N+T_{M}-q \\ I D X(P T)-q\end{array}\right)=\sum A_{q}\left(\begin{array}{c}1+2 M-1-q \\ 2 M-q\end{array}\right)=\sum A_{q}$

$\stackrel{A_{0}=0}{\longrightarrow} \sum_{q=1}^{q=M} H_{2}(\cdots, q) \stackrel{8.3)}{\longrightarrow}$

$\left.=\sum_{C=0}^{C=M-1}(-1)^{M-1-C} H_{1}([2, \cdots, M],[3, \cdots, 2 M-1], C) \stackrel{4.13)}{\longrightarrow} 1\right)$

$\left.S_{2}(M+2,2)=2^{M+1}-1=\operatorname{SUM}(2,[1, \cdots, 1],[1, \cdots, 2 M-1]) \rightarrow 2\right)$

q.e.d.

Example 8.1:

$$
\begin{aligned}
& M=1: 1=1 \\
& M=2: 1 \times 3-1 \times 2=1 \\
& M=3: 1 \times 3 \times 5-(1 \times 3 \times 4+1 \times 2 \times 4)+1 \times 2 \times 3=1 \\
& M=4: 1 \times 3 \times 5 \times 7-(1 \times 3 \times 5+1 \times 3 \times 4+1 \times 2 \times 4) \times 6 \\
& \quad+(1 \times 2 \times 3+1 \times 3 \times 4+1 \times 2 \times 4) \times 5-1 \times 2 \times 3 \times 4=1 \\
& M=5: 1 \times 3 \times 5 \times 7 \times 9-(1 \times 3 \times 5 \times 7+1 \times 3 \times 4 \times 6+1 \times 2 \times 4 \times 6+1 \times 3 \times 5 \times 6) \times 8 \\
&+(1 \times 2 \times 3 \times 5+1 \times 3 \times 4 \times 5+1 \times 2 \times 4 \times 5+1 \times 3 \times 5 \times 6+1 \times 2 \times 4 \times 6 \\
&+1 \times 3 \times 4 \times 6) \times 7-(1 \times 2 \times 3 \times 4+1 \times 2 \times 3 \times 5+1 \times 2 \times 4 \times 5+1 \times 3 \times 4 \times 5) \times 6 \\
&+1 \times 2 \times 3 \times 4 \times 5=1
\end{aligned}
$$

It can be concluded from the definition:

$$
\begin{aligned}
& \text { 8.5) } H_{2}([1, \cdots, 1],[1, \cdots, M], C)=(-1)^{M-C} C ! E_{M-C}^{C}=(-1)^{M-C} C ! S_{2}(M, C) \\
& N^{M}=\nabla \operatorname{SUM}(N,[1, \cdots, 1],[1, \cdots, M]) \\
& \stackrel{\mathrm{Form}_{2}}{\longrightarrow} \nabla \sum A_{q}\left(\begin{array}{c}
N+T_{M}-q \\
I D X(P T)-q
\end{array}\right)=\sum A_{c}\left(\begin{array}{c}
N+M-(M-C)-1 \\
M+1-(M-C)-1
\end{array}\right) \\
& =\sum A_{c}\left(\begin{array}{c}
N+C-1 \\
C
\end{array}\right) \\
& \text { 8.6) } N^{M}=\sum_{K=0}^{M} K ! S_{2}(M, K)\left(\begin{array}{c}
N \\
K
\end{array}\right)=\sum_{K=0}^{M}(-1)^{M-K} K ! S_{2}(M, K)\left(\begin{array}{c}
N+K-1 \\
K
\end{array}\right)
\end{aligned}
$$

It can be concluded from the definition:

$$
\begin{aligned}
& \text { 8.7) } H_{2}([1+y, 2+y, \cdots, M+y],[1,2, \cdots, M], C)=C ![y]^{M-C}\left(\begin{array}{c}
M \\
C
\end{array}\right) \\
& {[x+y]^{M}=\nabla \operatorname{SUM}(x,[1+y, 2+y, \cdots, M+y])} \\
& \stackrel{\text { Form }_{2}}{\longrightarrow} \nabla \sum_{c=0}^{M}\left(\begin{array}{c}
M \\
c
\end{array}\right) C ![y]^{M-c}\left(\begin{array}{l}
x+M-(M-C) \\
M+1-(M-C)
\end{array}\right)
\end{aligned}
$$




$$
\begin{aligned}
& =\nabla \sum_{c=0}^{M}\left(\begin{array}{c}
M \\
c
\end{array}\right) c ![y]^{M-c}\left(\begin{array}{c}
x+C \\
C+1
\end{array}\right)=\sum_{c=0}^{M}\left(\begin{array}{c}
M \\
c
\end{array}\right) c ![y]^{M-c}\left(\begin{array}{c}
x+C-1 \\
C
\end{array}\right) \\
& =\sum_{K=0}^{M}\left(\begin{array}{c}
M \\
K
\end{array}\right)[y]^{M-K}[x+k-1]_{K}=\sum_{K=0}^{M}\left(\begin{array}{c}
M \\
K
\end{array}\right)[y]^{M-K}[x]^{K} \rightarrow \text { Norlund identity }
\end{aligned}
$$

\section{Form 3 and Eulerian Number}

Rewrite (1) of section 3 as

$\sum_{n=0}^{N-1} n\left(\begin{array}{c}n+K \\ M\end{array}\right)=(M-K)\left(\begin{array}{c}N+K+1 \\ M+2\end{array}\right)+(1+K)\left(\begin{array}{c}N+K \\ M+2\end{array}\right)$

$P S=\left[K_{1}: D_{1}, \cdots, K_{M}: D_{M}\right], P T=\left[T_{1}, \cdots, T_{M}\right]$, use the same method of 3.1) to prove:

9.1) Use the Form $_{3}=\left(T_{1}+K_{1}\right) \cdots\left(T_{M}+K_{M}\right)=\sum X_{1} \cdots X_{M}$,

$\operatorname{SUM}(N, P S, P T)=\sum A_{q}\left(\begin{array}{c}N+T_{M}-q \\ I D X(P T)\end{array}\right), q=$ count of $X \in T . A_{q}=\prod_{i=1}^{M} B_{i}$,

$B_{i}=\left\{\begin{array}{l}-K_{i}+\left[T_{i}-m\right] D_{i} ; X_{i}=T_{i}, m=\text { count of }\left\{\cdots, X_{i-1}\right\} \in T \\ K_{i}+m D_{i} ; X_{i}=K_{i}, m=\text { count of }\left\{\cdots, X_{i-1}\right\} \in T\end{array}\right.$

Define $H_{3}(P S, P T, C)=A_{q}$ of 9.1), $C=$ Count of $X \in T$

$\left\langle\begin{array}{c}M \\ k\end{array}\right\rangle$ is Eulerian number. Worpitzky identity: $N^{M}=\sum_{k=0}^{M-1}\left\langle\begin{array}{c}M \\ k\end{array}\right\rangle\left(\begin{array}{c}N+k \\ M\end{array}\right)$

Already known 1) $\left\langle\begin{array}{c}M \\ M-q-1\end{array}\right\rangle=\left\langle\begin{array}{c}M \\ k\end{array}\right\rangle$,

2) $\left\langle\begin{array}{c}M \\ q\end{array}\right\rangle=(M-q)\left\langle\begin{array}{c}M-1 \\ q-1\end{array}\right\rangle+(q+1)\left\langle\begin{array}{c}M-1 \\ q\end{array}\right\rangle$

9.2) $H_{3}(P T, P T, q>0)=0 ; H_{3}(P T, P T, 0)=\prod T_{i} \quad$ This $\left.\rightarrow 4.5\right)$

9.3) $H_{3}([1, \cdots, 1],[1, \cdots, M], q)=\left\langle\begin{array}{c}M \\ M-q-1\end{array}\right\rangle=\left\langle\begin{array}{c}M \\ q\end{array}\right\rangle$,

$H_{3}([0, \cdots, 0],[1, \cdots, M], q)=\left\langle\begin{array}{c}M \\ q-1\end{array}\right\rangle$

[Proof]

Obviously: $H_{3}\left(\left[K_{1}=1, \cdots, K_{M}\right],\left[T_{1}=1, \cdots, T_{M}\right], M\right)=0$

$$
\begin{array}{r}
N^{M}=\operatorname{\nabla SUM}(N,[1, \cdots, 1])=\sum_{q=0}^{M} A_{q}\left(\begin{array}{c}
N+M-q-1 \\
M
\end{array}\right) \\
=\sum_{q=0}^{M} A_{q}\left(\begin{array}{c}
N+M-q-1 \\
M
\end{array}\right)=\sum_{k=0}^{M-1}\left(\begin{array}{c}
M \\
k
\end{array}\right)\left(\begin{array}{c}
N+k \\
M
\end{array}\right)
\end{array}
$$

q.e.d.

It's easy to deduce:

$\left.{ }^{*}\right) H_{3}(q)=H_{2}([0, \cdots, 0],[1, \cdots, M], q)=\sum \prod B_{i}$,

$B_{i}=\left\{\begin{array}{l}m+1: X_{i}=T_{i}, m=\text { count of }\left\{\cdots, X_{i-1}\right\} \in K \\ m: X_{i}=K_{i}, m=\text { count of }\left\{\cdots, X_{i-1}\right\} \in T\end{array}\right.$

$\left.\left({ }^{\star} 1\right) H_{3}(q)=H_{2}(M-q-1) \rightarrow 1\right)$

$\left.\left({ }^{\star} 2\right) \quad H_{3}(q)=(M-q) H_{3}(q-1)+(q+1) H_{3}([0, \cdots, 0],[1, \cdots, M-1], q) \rightarrow 2\right)$ 
(*3) $H_{3}(q)=\sum \prod B_{i}$, then $B_{1}+B_{2}+\cdots+B_{M}=q(M-q+1)$

$H_{3}([1, \cdots, 1],[1, \cdots, M], 1)$

$=1^{M-1} \times(M-1)+1^{M-2} \times(M-2) \times 2+1^{M-3} \times(M-3) \times 2^{2}+\cdots$

$=2^{0} \times M+2^{1} \times M+\cdots+2^{M-2} \times M-\left(2^{0} \times 1+2^{1} \times 2+\cdots+2^{M-2} \times(M-1)\right)$

$=M\left(2^{M-1}-1\right)-\sum_{i=0}^{M-2} 2^{i}(i+1)=M\left(2^{M-1}-1\right)-\left(2^{M-1}-1\right)-\sum_{i=0}^{M-2} 2^{i} i$

$=\left\langle\begin{array}{c}M \\ 1\end{array}\right\rangle=2^{M}-(M+1)$, the final equation is a known property of $\left\langle\begin{array}{c}M \\ 1\end{array}\right\rangle \rightarrow$

9.4) $\sum_{i=0}^{M} 2^{i} i=(M-1) 2^{M+1}+2$

9.5) $\left\langle\begin{array}{c}M \\ q\end{array}\right\rangle=\sum_{t_{1}+\cdots+t_{q+1}=M-q-1} 1^{t_{1}} 2^{t_{2}} \cdots(q+1)^{t_{q+1}}\left(1+t_{1}\right) \cdots\left(1+t_{1}+\cdots+t_{q}\right), t_{i} \geq 0$

[Proof]

$H_{3}([0, \cdots, 0],[1, \cdots, M], q+1)=\left\langle\begin{array}{c}M \\ q\end{array}\right\rangle=\sum \Pi(X \in T) \Pi(X \in K)$

If $X \in K$ is certain, then $X \in T$ is certain and $X_{1} \cdots X_{M}$ is certain.

When $X_{1} \cdots X_{M}>0$, then $X_{1}=T_{1}=1, K_{i} \in[1, q+1]$

Record $\Pi(X \in K)=1^{t_{1}} 2^{t_{2}} \cdots(q+1)^{t_{q+1}}, t_{1}+t_{2}+\cdots+t_{q+1}=M-q-1$

Take out factors $>0$, record as $\left.\left\{P_{1}, P_{2}, \cdots\right\},{ }^{*}\right) \rightarrow$

$\Pi(X \in T)=1^{P_{1}}\left(1+t_{p 1}\right)^{P_{2}-P_{1}}\left(1+t_{p 1}+t_{p 2}\right)^{P_{3}-P_{2}} \ldots$, it can be rewritten as

$\Pi(X \in T)=\left(1+t_{1}\right)\left(1+t_{1}+t_{2}\right)\left(1+t_{1}+t_{2}+t_{3}\right) \cdots\left(1+t_{1}+\cdots+t_{q}\right)$

q.e.d.

This is the conclusion of [7], which is obtained by guess and proved by induction.

Example 9.1:

$$
\begin{aligned}
& \left\langle\begin{array}{l}
6 \\
2
\end{array}\right\rangle=\sum_{t_{1}+t_{2}+t_{3}=3} 1^{t_{1}} 2^{t_{2}} 3^{t_{3}}\left(1+t_{1}\right)\left(1+t_{1}+t_{2}\right) \\
& =1^{3} 2^{0} 3^{0}(1+3)(1+3+0)+1^{2} 2^{1} 3^{0}(1+2)(1+2+1)+1^{2} 2^{0} 3^{1}(1+2)(1+2+0) \\
& +1^{1} 2^{1} 3^{1}(1+1)(1+1+1)+1^{1} 2^{2} 3^{0}(1+1)(1+1+2)+1^{1} 2^{0} 3^{2}(1+1)(1+1+0) \\
& +1^{0} 2^{1} 3^{2}(1+0)(1+0+1)+1^{0} 2^{2} 3^{1}(1+0)(1+0+2) \\
& +1^{0} 2^{3} 3^{0}(1+0)(1+0+3)+1^{0} 2^{0} 3^{3}(1+0)(1+0+0) \\
& =302
\end{aligned}
$$

\section{Conflicts of Interest}

The author declares no conflicts of interest regarding the publication of this paper.

\section{References}

[1] Peng, J. (2020) Shape of Numbers and Calculation Formula of Stirling Numbers. Open Access Library Journal, 7: e6081. https://doi.org/10.4236/oalib.1106081

[2] Peng, J. (2020) Subdivide the Shape of Numbers and a Theorem of Ring. Open Access Library Journal, 7: e6719. https://doi.org/10.4236/oalib.1106719

[3] Peng, J. (2020) Subset of the Shape of Numbers. Open Access Library Journal, 7: 
e7040. https://doi.org/10.4236/oalib.1107040

[4] Peng, J. (2021) Expansion of the Shape of Numbers. Open Access Library Journal, 8: e7120. https://doi.org/10.4236/oalib.1107120

[5] Editorial Board of Handbook of Modern Applied Mathematics (2002) Handbook of Modern Applied Mathematics Discrete Mathematics Volume. Tsinghua University Press, Beijing.

[6] Wang, Y.K. and Li, S.X. (1999) The Uniform Expression for Two Kinds of Stirling Number and Bernoulli Number. Journal of Guangxi Teachers College (Natural Science Edition), 1, 49-53.

[7] Qi, D.-J. (2012) A New Explicit Expression for the Eulerian Numbers. Journal of Qingdao University of Science and Technology (Natural Science Edition), 33, 439-440. 